\title{
Simultaneous Temperature and Deformations Measurements Using Long-Wave Infrared Speckle Interferometry: A Novel Hybrid Technique for Industrial Nondestructive Testing
}

\author{
Marc Georges ${ }^{1}$, Jean-François Vandenrijt ${ }^{1}$, Cédric Thizy ${ }^{1}$, Igor Alexeenko ${ }^{2,6}$, \\ Giancarlo Pedrini ${ }^{2}$, Jonathan Rochet ${ }^{3}$, Birgit Vollheim ${ }^{4}$, Iagoba Jorge ${ }^{5}$, \\ Pablo Venegas ${ }^{5}$, Ion Lopez ${ }^{5}$, and Wolfgang Osten ${ }^{2}$ \\ ${ }^{1}$ Centre Spatial de Liège, Univ. de Liège, Av. du Pré Aily, B-4031, Angleur, Belgium \\ mgeorges@ulg.ac.be \\ ${ }^{2}$ Institut für Technische Optik - Univ.Stuttgart, Pfaffenwaldring 9, 70569 Stuttgart, \\ Germany \\ ${ }^{3}$ Optrion, PIMW, Boulevard de Colonster 4, B-4000 Liège, Belgium \\ ${ }^{4}$ InfraTec GmbH, Gostritzer Str. 61-63, 01217 Dresden, Germany \\ ${ }^{5}$ Centro de Tecnologías Aeronáuticas, Parque Tecnológico de Álava, Miñano 01510, Spain \\ ${ }^{6}$ Immanuel Kant Baltic Federal University 236041, Kaliningrad, \\ A.Nevskogo str. 14, Russia
}

\section{Introduction}

Since its demonstration in 2003 [1], the use of long-wave infrared (LWIR) $\mathrm{CO}_{2}$ lasers in Digital Holography (DH) has been shown in an increasing number of applications. In particular it allows observing large objects and in metrology it allows measuring large displacements while rendering holography more immune to environmental perturbations, compared to holography in the visible. For metrology we demonstrated various holographic techniques in LWIR: speckle interferometry [2,4], DH interferometry [2-4] and shearography [4]. The LWIR holographic set-up generally makes use of microbolometer arrays based thermal imagers. The present paper discusses some result of the European project FANTOM in which another advantage was put forward when speckle interferometry is applied in LWIR: the thermal background is captured simultaneously to the specklegrams since it constitutes an offset in the signal. Therefore one can correlate uniquely the temperature and displacement information at the same time and in each pixel. We describe here the successful achievements of the project.

\section{Simultaneous Temperature and Deformation Measurements}

Since the aim of our project was to have the thermal background in addition to the spatially varying information coming from the object and reference beam, the only 
technique which is viable is Electronic Speckle Pattern Interferometry (ESPI) instead of digital holographic interferometry. Indeed the thermal background of the signal arises from incoherent infrared light emitted by the object, whereas the local interference (specklegram) is only present when the laser is illuminating the object. Observing both signals at the same time is only possible if the scene is directly imaged by the thermal camera through a lens, and not reconstructed through a lensless digital holography technique.

\section{LWIR Electronic Speckle Pattern Interferometry}

The development of the ESPI set-up has been presented already [4]. A mobile system was built for measurements in field conditions and was also presented recently [5]. Fig. 1 shows the instrument and its construction. It is constituted by a lower bench (Fig.1(a)) with the laser and beam separator which is a stack of Brewster angle plates in a chevron geometry and which separate 2 components of polarization (Fig.1(b)). The transmitted object beam travels to the object through a periscope. The reflected beam (reference beam) travels to an upper bench (Fig.1(c)) is transformed prior to be made incident to the camera through a large beam combiner (BC). The separation between both signals can be achieved by recording the thermal image separately in a first step by closing the laser shutter. Shortly after the shutter is open to record the specklegram (interference of object and reference beams). The specklegram is processed by phase-shifting in order to retrieve the phase at this instant. This operation is performed at various instants during the phenomena observation and the difference between specklegrams is computed to show the deformation, while the difference in thermograms shows the simultaneous temperature variations.

(a)

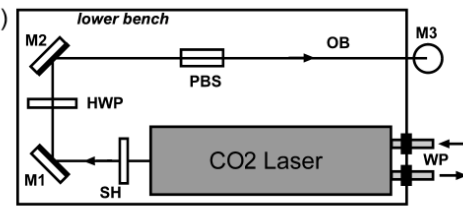

(c)

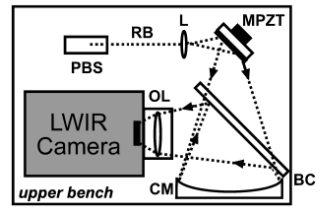

(b)

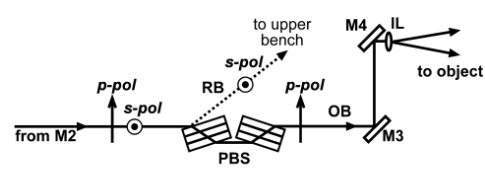

PBS: Polarizing Beamsplitter $\mathbf{B C}$ : Beam Combiner SH: Shutter $\quad$ CM : Concave Mirror L: Lens
IL: Illuminating Lens OL: Objective Lens $\quad$ OB : Object Beam OL: Objective Lens $\quad$ MPZT: Mirror + Piezo-element Mi: Mirrors

WP : Water Pipes

Fig. 1 Scheme of the mobile LWIR ESPI system: (a) lower bench with the laser and beam separation, (b) the principle of beam separation by Brewster angle plates in chevron geometry, (c) the upper bench with the beam combiner and the camera 


\section{$4 \quad$ Typical Results and Applications}

A typical result is shown in Fig.2 and concerns detection of defects inside a composite aeronautical structure made of a sandwich structure with CFRP skins glued on foam core and defects consisting of lack of glue (in grey) and cracked core (in black) (Fig.2(a)). Fig.2(b) shows the interferogram obtained by differentiating two specklegrams obtained before and after heating with a halogen lamp. The defects are seen as local variations inside the global quadratic deformation. After processing (phase unwrapping and removal of global deformation by polynomial fit, Fig.2(c) is obtained and shows the defects. This image can be compared to similar results obtained in the same conditions by a separate shearographic instrument (Fig.2 (f)). On another hand, the thermograms difference captured at the same instants than the specklegram gives the thermographic image in Fig.2(e). The latter is compared to classical thermography result shown in Fig.2(d), that was obtained with a separate active thermography equipment with modulated lamp and the optical lock-in (OLT) method.

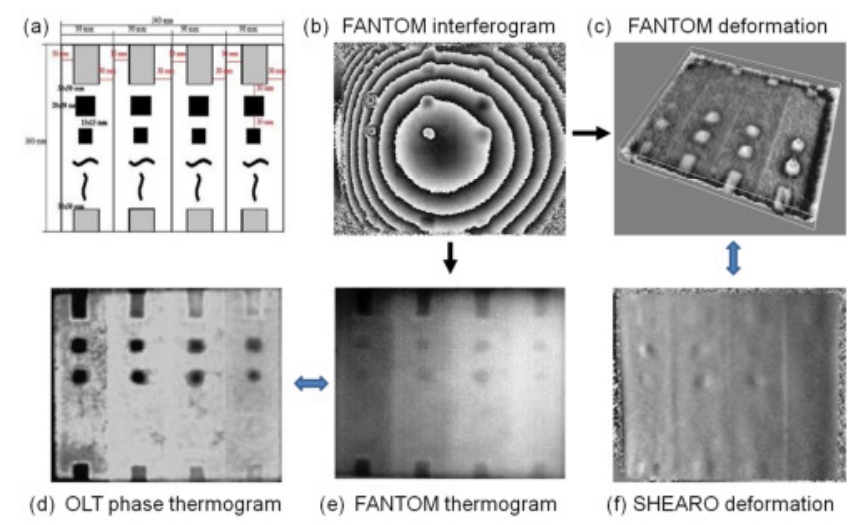

Fig. 2 Defect detection capabilities with the FANTOM technique compared to separate thermography and shearography

Another interesting example is shown in Fig.3 where a large composite structure was examined in field conditions. It has several defects included and the structure was heated by halogen lamps. The two parts of the signals are shown. The deformation (phase variation) shows a deep defect (marked by a square) that the temperature part doesn't and conversely where a defect (marked by a circle) is better seen with temperature. This shows the interest of our method which improves the probability of detecting defects compared to separate techniques. 

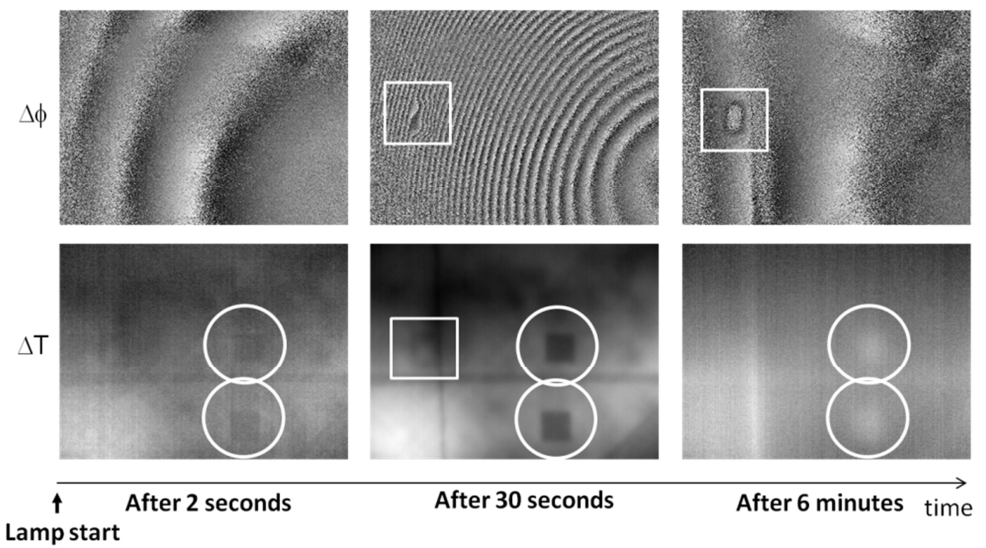

Fig. 3 Deformation $(\Delta \phi)$ and temperature variations $(\Delta \mathrm{T})$ during a sequence of heating a composite structure. Each signal show different defects.

\section{Conclusions}

ESPI in the Long Wave IR was demonstrated as a mean to record simultaneously the temperature variations and deformation. It can be used in defects detection where thermography and holography/shearography often appear as complementary methods. Here the new technique is used to improve the probability of detecting defects when compared to separate techniques. This work has been carried out in the frame of the EU funded FANTOM project (contract 213457).

\section{References}

1. Allaria, E., Brugioni, S., De Nicola, S., Ferraro, P., Grilli, S., Meucci, R.: Digital holography at $10.6 \mu \mathrm{m}$. Optics Communication 215, 257-262 (2003)

2. Vandenrijt, J.-F., Georges, M.P.: Electronic speckle pattern interferometry and digital holographic interferometry with microbolometer arrays at $10.6 \mu \mathrm{m}$. Applied Optics 49(27), 5067-5075 (2010)

3. Alexeenko, I., Vandenrijt, J.-F., Georges, M.P., Pedrini, G., Cédric, T., Osten, W., Vollheim, B.: Digital holographic interferometry by using long wave infrared radiation $\left(\mathrm{CO}_{2}\right.$ Laser). Applied Mechanics and Materials 24-25, 147-152 (2010)

4. Alexeenko, I., Vandenrijt, J.-F., Pedrini, G., Thizy, C., Vollheim, B., Osten, W., Georges, M.P.: Nondestructive testing by using long-wave infrared interferometric techniques with $\mathrm{CO} 2$ lasers and microbolometer arrays. Applied Optics 52(1), A56-A67 (2013)

5. Vandenrijt, J.-F., Thizy, C., Alexeenko, I., Pedrini, G., Rochet, J., Vollheim, B., Jorge, I., Venegas, P., Lopez, I., Osten, W., Georges, M.P.: Mobile speckle interferometer in the long-wave infrared for aeronautical nondestructive testing in field conditions. Optical Engineering 52(10), 101903 (2013) 